
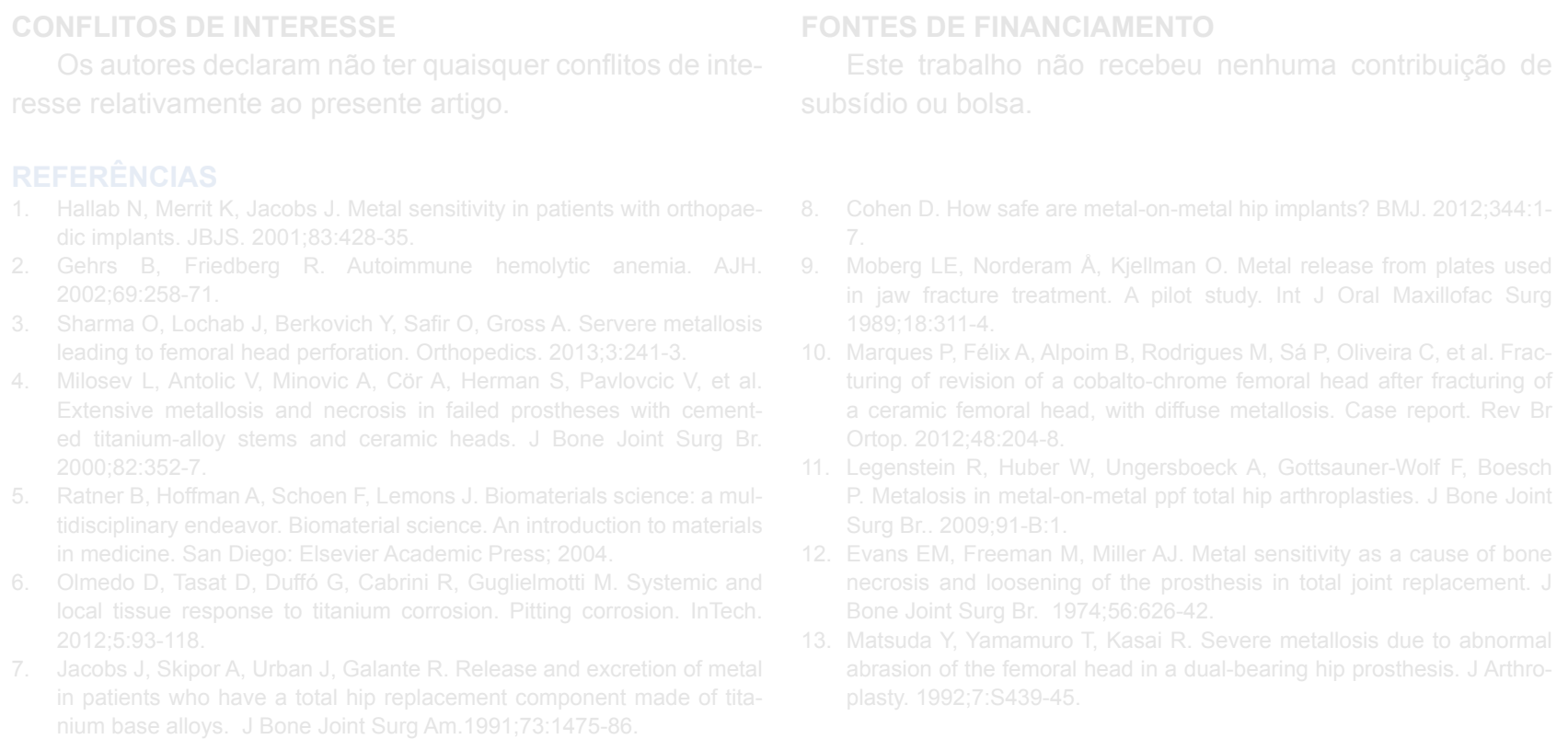

\title{
Intoxicação por Supervarfarina
}

\author{
Superwarfarine Poisoning
}

\author{
Ana FREIXO $\rrbracket^{1}$, Luís LOPES ${ }^{2}$, Manuela CARVALHO ${ }^{1}$, Fernando ARAÚJO ${ }^{1,3}$ \\ Acta Med Port 2015 May-Jun;28(3):389-392
}

\section{RESUMO}

Os raticidas do tipo supervarfarina são usados em todo o mundo e distinguem-se da varfarina pela sua elevada potência e duração de acção anticoagulante. O fácil acesso a este produto possibilita o seu uso no envenenamento humano acidental ou deliberado. É apresentado um caso clínico de intoxicação voluntária de raticida (RATIBRON ${ }^{\circledR}$ ) por uma mulher que ingeriu uma quantidade total estimada de 27,5 mg de bromadiolona durante duas semanas, com manifestações hemorrágicas ligeiras, cuja reversão do efeito anticoagulante, com correç̧ão do estudo da coagulação, demorou cerca de um mês. A correcção dos valores da hemostase é habitualmente demorada e não existem orientações terapêuticas para estes casos, sendo que uma diminuição gradual das doses de vitamina $\mathrm{K}$, em ambulatório, acompanhada do estudo do International Normalized Ratio, permite avaliar com segurança a resposta à terapêutica. Palavras-chave: Coagulopatias; Rodenticidas/intoxicação; Vitamina K.

\section{ABSTRACT}

The superwarfarin-type anticoagulant rodenticides are used throughout the world and distinguish themselves from warfarin for its high potency and long acting anticoagulant activity. Easy access to these products enables the accidental or deliberate human poisoning. A case of voluntary rodenticide poisoning (RATIBRON ${ }^{\circledR}$ ) by a woman who ingested an estimated $27.5 \mathrm{mg}$ of bromadiolone total quantity for two weeks, with minor bleeding episodes, whose reversal of the anticoagulant effect with the correction of the abnormal values of the clotting tests took about one month to reverse is reported here. The correction of the haemostasis defects takes usually a long time and there are no treatment guidelines, but a gradually vitamin $\mathrm{K}$ dosage reduction, as out patients, along with the monitoring of the International Normalized Ratio levels, allows a safe evaluation of the therapeutic response.

Keywords: Blood Coagulation Disorders; Rodenticides/poisoning; Vitamin K.

\section{INTRODUÇÃO}

Os anticoagulantes dicumarínicos foram inicialmente utilizados como raticidas, tendo posteriormente sido aperfeiçoados no sentido de aumentar a sua eficácia através

de uma duração de acção mais longa e de uma maior potência, sendo denominados de supervarfarinas. A alta eficácia deve-se a uma maior solubilidade lipídica e superior

1. Serviço de Imuno-hemoterapia. Centro Hospitalar de São João. Porto. Portugal.

2. Serviço de Medicina Intensiva. Unidade Polivalente de Cuidados Intermédios Geral. Centro Hospitalar de São João. Porto. Portugal.

3. Departamento de Medicina. Faculdade de Medicina. Universidade do Porto. Porto. Portugal.

$\bowtie$ Autor correspondente: Ana Freixo. ana.parente.freixo@gmail.com

Recebido: 21 de Agosto de 2014 - Aceite: 04 de Fevereiro de 2015 | Copyright @ Ordem dos Médicos 2015 
afinidade pelo tecido hepático, provocando uma eliminação em duas fases, sendo que no caso da bromadiolona consiste numa primeira mais rápida (semi-vida de seis dias) e numa fase seguinte com duração de cerca de 24 dias. $^{1}$

A bromadiolona é um raticida usado mundialmente. Em ratos, é três vezes mais potente do que a varfarina, mas a sua toxicidade em humanos é ainda desconhecida. ${ }^{1} \mathrm{O}$ fácil acesso a este produto possibilita o seu uso no envenenamento humano acidental ou deliberado, provocando uma coagulopatia prolongada com risco hemorrágico elevado. Felizmente, a maioria dos casos descritos não são letais, ${ }^{2-4}$ sublinhando o papel importante de uma história clínica completa que é a chave para um diagnóstico e tratamento atempados, já que a maioria dos hospitais não possui análises laboratoriais capazes de identificar ou dosear estes fármacos.

O tratamento tradicional é a administração de vitamina $\mathrm{K}$ de forma empírica, já que não existem recomendações quer quanto à dose, quer quanto ao número de administrações eficazes na reversão deste tipo de coagulopatias, ou mesmo em relação ao período em que estes doentes devem ser seguidos de forma a assegurar que a reversão foi completa.

Apresenta-se um caso clínico de tentativa de suicídio por envenenamento com bromadiolona.

\section{CASO CLÍNICO}

Trata-se de uma doente do sexo feminino, de 46 anos de idade, com antecedentes de síndrome depressivo/ansioso, que se dirigiu ao serviço de urgência do Centro Hospitalar de São João por ingestão voluntária de uma quantidade total aproximada de $27,5 \mathrm{mg}$ de bromadiolona (RATIBRON ${ }^{\circledR}$ ) $(0,44 \mathrm{mg} / \mathrm{kg})$ nas duas últimas semanas, dos quais $10,5 \mathrm{mg}$ $(0,17 \mathrm{mg} / \mathrm{kg})$ nas últimas quatro horas. Nos cinco dias anteriores, apresentava episódios de epistáxis espontâneas e metrorragias, com perdas ligeiras de sangue. Sem outras queixas. Habitualmente medicada com fluoxetina $20 \mathrm{mg} /$ dia, alprazolam $20 \mathrm{mg} / \mathrm{dia}$, diazepam $10 \mathrm{mg} / \mathrm{dia}$. Sem história pessoal ou familiar de coagulopatias hereditárias.

Ao exame objectivo apresentava-se visivelmente ansiosa, pálida, hidratada, anictérica e hemodinamicamente estável (dados antropométricos: $62 \mathrm{~kg}$ de peso e 1,65 $\mathrm{m}$ de altura). Sem sinais de hemorragia activa e restante exame objectivo sem alterações.

O estudo analítico revelou ligeira anemia, com 11,1 g/ $\mathrm{dL}$ de hemoglobina $(\mathrm{Hb})$, estudo da coagulação com tempo de tromboplastina parcial activado (aPTT) de 54,9 segundos (valores de referência: 24,5 a 36,5 segundos), tempo de protrombina (TP) de 96,8 segundos (valores de referência: 10,0 a 14,0 segundos), International Normalized Ratio (INR) de 8,00 e doseamento dos factores II, VII e X (PeP) $<5 \%$. Marcadores de função hepática sem alterações. $O$ despiste de drogas na urina foi positivo apenas para benzodiazepinas.

O tratamento de urgência incluiu lavagem gástrica com administração de $50 \mathrm{~g}$ de carvão activado, $10 \mathrm{mg}$ de vitamina $\mathrm{K}$ endovenosa e $1000 \mathrm{UI}$ de concentrado de complexo protrombínico (CCP), com controlo da clínica hemorrágica e correção parcial do estudo da coagulação, com aPTT 40,0 segundos (valores de referência: 24,5 a 36,5 segundos), PT 19,5 segundos (valores de referência: 10,0 a 14,0 segundos), PeP $31 \%$ e INR 1,48.

Decidiu-se o internamento na Unidade de Cuidados Intermédios para vigilância de novos fenómenos hemorrágicos, controlo dos parâmetros da coagulação e avaliação psiquiátrica. Durante este período foi administrada vitamina $\mathrm{K}$ por via oral, uma toma diária, na dose de $30 \mathrm{mg}$, apresentando valores de INR entre 1,26 e 1,64. A doente teve alta ao terceiro dia de internamento sem registo de novos episódios hemorrágicos e com valor de INR de 1,30. Contudo, atendendo à longa semi-vida da bromadiolona, foi orientada para seguimento em regime de hospital de dia de Imuno-hemoterapia (HDI).

Na primeira consulta (03/06/2014) apresentava um valor de INR de 4,91, tendo sido medicada com vitamina $\mathrm{K}$, $30 \mathrm{mg}$ uma toma diária, por via oral, durante uma semana. Na segunda consulta (11/06/2014) apresentava INR de 2,55 e por intolerância à terapêutica oral, queixando-se de náuseas e vómitos aquando das tomas, foi decidido alterar a via de administração para endovenosa, $20 \mathrm{mg}$ uma toma por dia, durante sete dias (tendo sido o tratamento diário efectuado no Centro de Saúde). O INR passou para 1,46 à terceira consulta $(18 / 06 / 2014)$ e foi medicada durante mais sete dias com vitamina $\mathrm{K}$, por via endovenosa, na dose de $10 \mathrm{mg}$, uma toma por dia. Após vinte e um dias (25/06/2014) de terapêutica em ambulatório apresentava um valor de INR de 0,96 e decidiu-se então suspender a administração de vitamina $\mathrm{K}$ e agendar nova consulta dentro de sete dias. Nessa altura (02/07/2014), o INR era de 1,48 , tendo sido administrados $10 \mathrm{mg}$ de vitamina $\mathrm{K}$ endovenosa e dada alta da consulta, após cerca de um mês de follow-up. Durante o período entre a alta do internamento e a alta da consulta externa, a doente referiu dois episódios hemorrágicos ligeiros e auto-limitados (epistáxis e gengivorragia), que não motivaram a sua vinda à urgência, e negou novas tentativas de intoxicação (Fig. 1).

\section{DISCUSSÃO}

Apresenta-se um caso de intoxicação voluntária de uma mulher que ingeriu uma quantidade total estimada de 27,5 $\mathrm{mg}$ de bromadiolona, durante duas semanas, cujo efeito anticoagulante demorou cerca de um mês a reverter. A elevada semi-vida da bromadiolona deve-se à sua capacidade de acumulação hepática e a uma eliminação bifásica. $\mathrm{O}$ tempo de semi-vida e a toxicidade da bromadiolona em humanos não estão ainda bem estudados, mas está descrito que doses de $0,17 \mathrm{mg} / \mathrm{Kg}$ podem causar alterações da coagulação durante cerca de quatro semanas. ${ }^{1,5}$

Em geral, o tratamento das hemorragias associadas à varfarina deve ser efectuado com administração de vitamina $\mathrm{K}$, mas relativamente a intoxicações por supervarfarina (tal como a bromadiolona) as orientações terapêuticas são escassas.

As terapêuticas complementares à vitamina $\mathrm{K}$, são o CCP e o plasma fresco congelado (PFC), utilizadas 


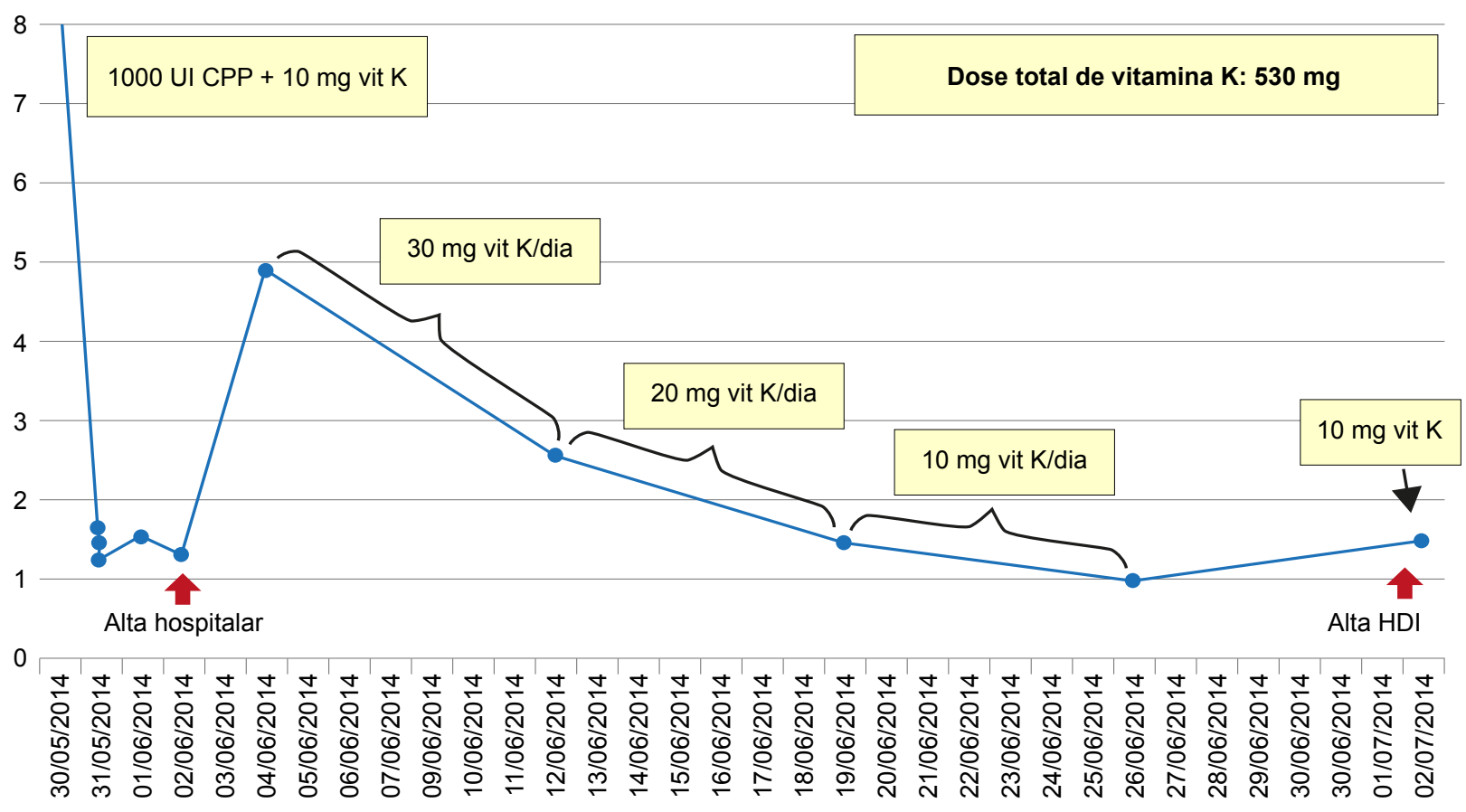

Figura 1 - Evolução temporal dos valores de INR

quando se pretende obter uma reversão rápida dos efeitos dos dicumarínicos, nomeadamente em caso de hemorragias graves (de notar que a acção da vitamina $\mathrm{K}$, mesmo quando usada por via endovenosa, demora cerca de 4 a 6 $\mathrm{h}$ até se obter um efeito relevante), sendo que a terapêutica de primeira linha, recomendada actualmente pelas normas internacionais é o CCP. ${ }^{6}$

Apesar de economicamente mais viável o PF apresenta muitas desvantagens, como a necessidade de administração de volumes elevados (10 - $15 \mathrm{~mL} / \mathrm{Kg}$, o que significa num adulto de $60 \mathrm{Kg}$, cerca de $900 \mathrm{~mL}$ ), possibilidade de ocorrência de reacções laterais graves (ex. TRALI) e tempo de processamento elevado (necessário determinar o grupo sanguíneo, descongelar as unidades de PFC e administrar o volume em causa, ou seja, poderá demorar 2 - $4 \mathrm{~h}$ até termos os efeitos desejados), para além da necessidade da sua disponibilidade. ${ }^{6}$ De sublinhar que tanto os factores da coagulação presentes no CCP como no PFC possuem semi-vidas curtas, pelo que em ambas as situações a administração concomitante de vitamina $\mathrm{K}$ é indispensável para tornar sustentável a correcção do efeito anticoagulante.

A administração inicial de CCP, no caso descrito, é extremamente discutível, pois não existindo clinica relevante, parece ter sido usada de forma preventiva (em função dos resultados laboratoriais), o que não é recomendado.

De salientar que o tratamento com vitamina K não é totalmente inofensivo, principalmente quando administrada por via endovenosa, ${ }^{7}$ mas a utilização da via oral pode trazer alguns problemas de adesão, como foi referido neste caso.

A vitamina $\mathrm{K}$, administrada diariamente a esta doente, corrigiu eficientemente a coagulopatia, mas possui, para este fim, uma semi-vida limitada e se a sua administração não for prolongada no tempo, considerando o tempo de acção da bromadiolona (total de 28 dias), podem reaparecer alterações da hemostase.

A avaliação do efeito da vitamina $\mathrm{K}$ e a decisão de suspender o tratamento foi efectuada indirectamente, em função do valor de INR e da avaliação da clínica hemorrágica, que nos pareceu uma abordagem simples e adequada. $A$ diminuição gradual e semanal das doses de vitamina K permitiu avaliar com maior segurança a resposta terapêutica.

\section{CONCLUSÃO}

De sublinhar que o doseamento no plasma dos antagonistas da vitamina $\mathrm{K}$ pressupõe uma análise laboratorial dispendiosa (cerca de 100 vezes mais cara do que a análise do INR) e morosa (demoram cerca de duas semanas a fornecer resultados), sendo que a disponibilidade de laboratórios para a realizar é também muito limitada (a única instituição, nas regiões Norte e Centro, que tenhamos conhecimento, que é capaz de efectuar o doseamento deste fármaco, em rotina, é o Instituto Nacional de Medicina Legal e Ciências Forenses, IP - delegação de Coimbra).

Infelizmente, não há dados sobre a incidência deste tipo de intoxicações em Portugal e tanto quanto é do nosso conhecimento, este será o primeiro caso publicado no nosso país de uma intoxicação por bromadiolona.

\section{PROTECÇÃO DE PESSOAS E ANIMAIS}

Os autores declaram que os procedimentos seguidos estavam de acordo com os regulamentos estabelecidos pelos responsáveis da Comissão de Investigação Clínica e Ética e de acordo com a Declaração de Helsínquia da Associação Médica Mundial. 


\section{CONFIDENCIALIDADE DOS DADOS}

Os autores declaram ter seguido os protocolos do seu centro de trabalho acerca da publicação dos dados de doentes.

\section{CONFLITOS DE INTERESSE}

Os autores declaram não terem quaisquer conflitos de interesse relativamente ao presente artigo.

\section{FONTES DE FINANCIAMENTO}

Este trabalho não recebeu qualquer contribuição de subsídio ou bolsa.

difethialone. Emergencias. 2013;25:201-3.

5. Hui CH, Lie A, Lam CK, Bourke C. Superwarfarin poisoning leading to prolonged coagulopathy. Forensic Sci Int. 1996;78:13-8.

6. Keelin D, Baglin T, Tait C, Watson H, Perry D, Baglin C, et al. Guidelines on oral anticoagulation with warfarin - fourth edition. $\mathrm{Br} \mathrm{J}$ Haematol. 2011;3:311-24.

7. Fiore LD, Scola MA, Cantillon CE, Brophy MT. Anaphylactoid reactions to vitamin K. J Thromb Thrombolysis. 2001;11:2:175-83. 
Ana FREIXO, Luís LOPES, Manuela CARVALHO, Fernando ARAÚJO

\section{Intoxicação por Supervarfarina}

Acta Med Port 2015:28:389-392

Publicado pela Acta Médica Portuguesa, a Revista Científica da Ordem dos Médicos

Av. Almirante Gago Coutinho, 151

1749-084 Lisboa, Portugal.

Tel: +351218428 215

E-mail: submissao@actamedicaportuguesa.com

www.actamedicaportuguesa.com

ISSN:0870-399X | e-ISSN: 1646-0758

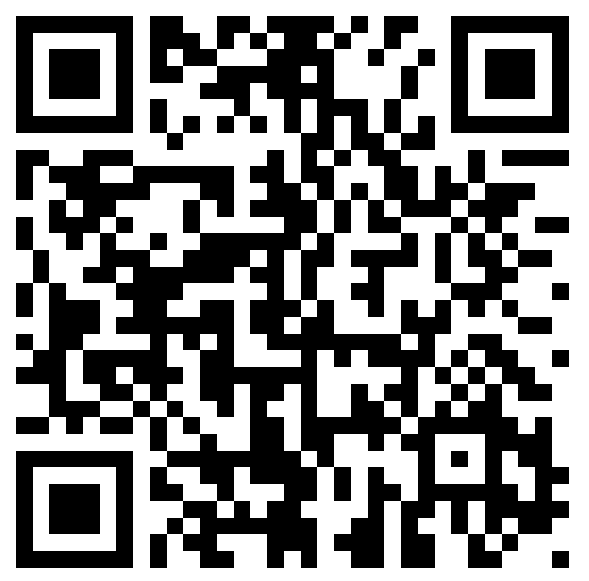

